\title{
Placenta percreta induced uterine rupture at 15 weeks of gestation: an unusual case
}

\author{
Sunita Samal ${ }^{*}$, Seetesh Ghose, P. Pallavee, Ramya Thulasi
}

Department of Obstetrics \& Gynecology, Mahatma Gandhi Medical College \& Research Institute, Pilliyarkuppam, Puducherry, India

Received: 27 September 2013

Accepted: 8 October 2013

\section{*Correspondence:}

Dr. Sunita Samal,

E-mail: sunisamal@rediffmail.com

(c) 2013 Samal S et al. This is an open-access article distributed under the terms of the Creative Commons Attribution Non-Commercial License, which permits unrestricted non-commercial use, distribution, and reproduction in any medium, provided the original work is properly cited.

\begin{abstract}
Spontaneous rupture of uterus during early pregnancy due to placenta percreta is a rare complication, which is potentially life-threatening to both mother \& fetus and also difficult to diagnose. A 27 years old pregnant woman with history of previous one caesarean section 2 years back was referred to our emergency department with severe anaemia and breathlessness. On examination patient was in hypovolemic shock. Ultrasonography revealed massive fluid collection in peritoneal cavity, an intrauterine anomalous foetus of 15 weeks and low-lying placenta. Paracentesis was positive for blood. On laparotomy there was haemoperitoneum of about 3 litres with placenta perforating through the lower segment of uterus. Even after all measures to conserve uterus, due to uncontrolled bleeding hysterectomy was performed. Postoperative period was uneventful. Pathological analysis of the specimen confirmed our diagnosis of placenta percreta. Though uterine rupture is rare in early trimester, it may be considered in a patient with haemoperitoneum with a scarred uterus, which can be due to placenta percreta also.
\end{abstract}

Keywords: Haemoperitoneum, Hysterectomy, Placenta percreta

\section{INTRODUCTION}

Placenta accreta is an abnormal deep attachment of placenta to the myometrium which is of two types: placenta increta and percreta. Placenta percreta is the rarest and most severe form. This is due to partial or total absence of decidua basalis, imperfect development of Nitabuch layer and firm adherence of placenta to uterine myometrium. ${ }^{1}$ In placenta accreta chorionic villi grow into the basal decidua, in increta it penetrate into the musculature and in placenta percreta the villi not only pass through the myometrium and serosa, but also penetrate adjacent structures like bladder and bowel resulting in catastrophic haemorrhage. Literature review shows spontaneous uterine rupture due to placenta percreta is rare and usually occurs in late second and third trimester. But its occurrence, resulting in life threatening situation, in first and early $2^{\text {nd }}$ trimester is very rare. Here we are reporting a case of a pregnant woman, who presented to us in haemorrhagic shock at $15 \mathrm{wks}$ of gestation due to placenta percreta induced uterine rupture.

\section{CASE REPORT}

A 27 years old pregnant woman (gravida 2, para 1) at 15 weeks of gestation with history of previous caesarean section was admitted to emergency department with breathlessness and pain abdomen for 6hours. On clinical examination she was in early hypovolemic shock with abdominal distension. Ultrasonography revealed gross fluid collection in peritoneal cavity with intrauterine 15 wks anomalous foetus and a low-lying placenta. Paracentesis was done to know the nature of fluid, which was positive for blood. A diagnosis of rupture uterus was made and planned for emergency laparotomy along with simultaneous resuscitation of the patient. On laparotomy there was haemoperitoneum of about 3litres, with $1-2 \mathrm{~cm}$ of rent at left anterior surface near isthmus through which 
placenta was protruding. The rent was extended transversely through which foetus with meningomyelocoele and part of the placenta was removed. Rest of the placenta was morbidly adherent to lower part of uterus. In view of unstable haemodynamic condition and uncontrolled bleeding from the placental site, total abdominal hysterectomy was performed to save the life of the woman. She received total 5units of whole blood. Her recovery was uneventful and was discharged on $8^{\text {th }}$ postoperative day. The final histopathological study revealed that the chorionic villi had invaded and extended deep into myometrium with absence of decidua basalis, confirming diagnosis of placenta percreta (Figure $1)$.

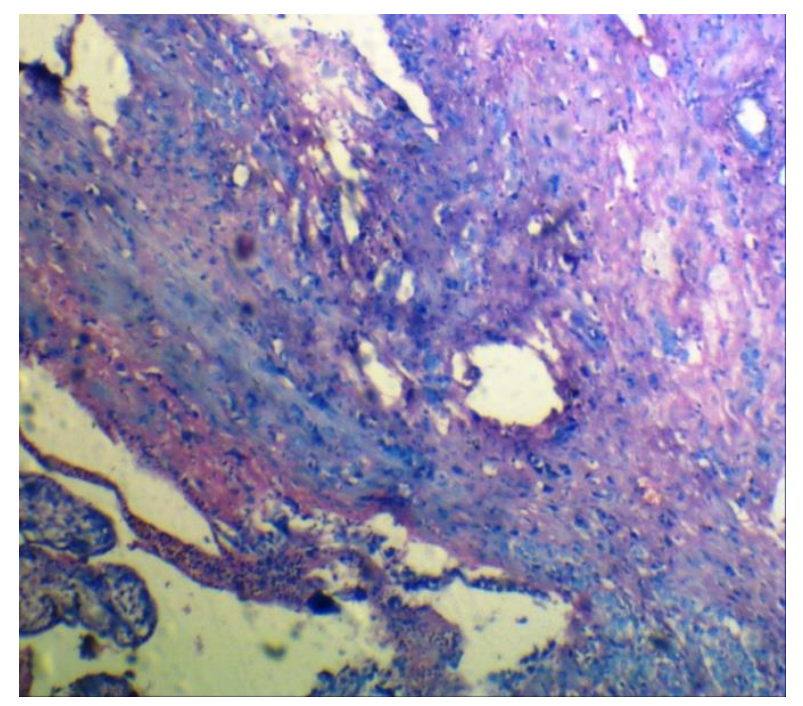

Figure 1: Showing chorionic villi invading and extending deep into the myometrium.

\section{DISCUSSION}

Placenta accreta occurs in approximately 1in 2500 deliveries (ACOG 2002). Stafford \& Belfort (2008) cite the incidence of accreta 1:2500 in 1980 to $1: 210$ in 2006, which is due to rising trend of caesarean sections. ${ }^{1}$ Placenta percreta is the rarest form with incidence of only 5\% among all accreta. Placenta percreta induced uterine rupture usually occurs during later part of pregnancy with only few cases reported in early trimester, as in our case. ${ }^{2}$ Literature review showed its occurrence as early as $6 \mathrm{wks}$ of gestation. ${ }^{3}$ Mostly uterine rupture during last trimester involves lower uterine segment and rupture in first trimester commonly affects fundus, but in our case it involved the lower pole of uterus. ${ }^{4}$ Predisposing factors for placenta accreta include prior dilatation and curettage, endometritis, high parity, previous uterine surgery, the most common being previous caesarean scar as in present case. Clark et al. showed in their study, the relationship of abnormal placentation and previous caesarean scar. As per this study with placenta praevia, probability of placenta accreta increases from $5 \%$ without prior section to $>24 \%$ with one and up to $67 \%$ with 4 or more prior caesarean. ${ }^{5}$ Wax et al, showed caesarean to conception intervals are shorter in patients with placenta accreta and in present case it was about 18 months. ${ }^{6}$ Monks et al. reported a case of gravida 4 para 3 with previous 3 sections with incomplete abortion at 10 wks of gestation. She underwent hysterectomy due to heavy bleeding which was unresponsive to conservative measures. Later histopathological analysis revealed a placenta percreta. ${ }^{7}$ Other reports concerned with placenta percreta are in higher gestational age. In pregnant patients with acute abdomen and features of intra-peritoneal fluid collection, differential diagnosis of appendicitis and haemoperitoneum should be considered. In case of haemoperitoneum the most probable diagnosis will be rupture uterus or invasive mole in early trimester. In patients with previous caesarean section, one should be aware of placenta praevia with placenta accreta. Generally the site of placenta percreta induced rupture will be more vascularised than the previous scar induced rupture, giving rise to massive haemoperitoneum as in our case. In placenta percreta the absence of spongious decidua leads to exposure of heavily perfused myometrial vessels with subsequent danger of haemorrhagic shock. So early diagnosis is of great importance. Conservative management of placenta accreta/ percreta can be successful and can preserve fertility. Management of these cases has varied, either with prophylactic or therapeutic uterine artery embolization or internal iliac ligation at the time of surgery. Some patients with placenta percreta being treated following delivery with methotrexate also. But several cases of delayed haemorrhage necessitating hysterectomy have been reported in such cases. In some cases there was no additional treatment after leaving placenta in situ. However, considering high mortality rate with conservative management of placenta percreta as compared to hysterectomy, the latter is preferred in emergency situation.

In conclusion, placenta percreta can lead to rupture of the uterus in early trimester resulting in catastrophic haemorrhage. Acute abdomen with haemoperitoneum in early trimester of pregnancy is a challenge to the obstetricians. Early diagnosis and prompt management can have successful outcome.

\section{REFERENCES}

1. Cunningham, Leveno, Bloom, Hauth, Rouse, Spong. Williams Obstetrics. $23^{\text {rd }}$ ed. New York; Mc Graw-Hill;2010. p776.

2. Park YJ, Ryu KY, Lee JI, Park MI. Spontaneous uterine rupture in the first trimester: a case report. J Korean Med Sci.2005;20:1079-81.

3. Ismail SI, Toon PG. First trimester rupture of previous caesarean section scar. J Obstet Gynaecol. 2007;27:202-4.

4. Schrinsky DC, Benson RC. Rupture of the pregnant uterus: a review. Obstet Gynecol Surv. 1978;33: 217-32. 
5. Clark SL. Koonings PP, Phelan JP. Placenta praevia accreta and prior cesarean section. Obstet. Gynecol.1985;66:89-92.

6. Wax JR, Seiler A, Horowitz S and Ingardia CJ. Interpregnancy interval as a risk factor for placenta accreta. Conn Med.2000;64:659-661.

7. Monks, P.L., Catalano, S. and Close, P.J. A case report: cervical pregnancy with placenta percreta and ultrasound assisted diagnosis. Asia-Oceania J. Obstet. Gynaecol.1993;19:37-41.

DOI: $10.5455 / 2320-1770 . i j r \operatorname{cog} 20131249$

Cite this article as: Samal S, Ghose S, Pallavee P, Thulasi R. Placenta percreta induced uterine rupture at 15 weeks of gestation: an unusual case. Int $\mathbf{J}$ Reprod Contracept Obstet Gynecol 2013;2:714-6. 